\title{
Análisis de las complicaciones de la cirugía de la hernia discal lumbar
}

\author{
P.A. Hernández-Pérez y H. Prinzo-Yamurri
}

Cátedra de Neurocirugía. Hospital de Clínicas Dr. Manuel Quintela. Universidad de la República Oriental del Uruguay

\section{Resumen}

Objetivo. Examinar los casos de hernia discal lumbar operados entre los años 1997 y 2002, realizando un análisis de las complicaciones quirúrgicas, a la vez que se realiza una revisión de la literatura en relación con el tema.

Material y métodos. Se revisaron las historias clínicas de 64 pacientes con diagnóstico de compresión radicular lumbar por hernia discal, sometidos a tratamiento quirúrgico, analizándose un total de 73 cirugías, incluyendo 9 reintervenciones realizadas en el período de tiempo definido al inicio.

Resultados. Se presentaron complicaciones en 7 procedimientos (9.6\%): 2 infecciones profundas, 1 caso de desgarro de la duramadre reparado, 2 casos de fístula de líquido cefalorraquídeo (LCR), 1 pseudomeningocele, y 1 caso de lesión de la raíz L5.

Conclusiones. Las complicaciones que se presentaron con mayor frecuencia fueron los desgarros de la duramadre y las infecciones, resultados que coinciden con las series internacionales publicadas.

PALABRAS CLAVE: Discectomía lumbar. Complicaciones. Cirugía espinal.

Analysis of the lumbar discectomy complications

\section{Summary}

Objective. To analyze the complications in a series of patients operated on for lumbar disc herniations between 1997 and 2002.

Methods. The records of 64 patients with the diagnosis of lumbar radicular compression by disc herniation who underwent 73 surgical procedures were analyzed. The patients with radicular pain without disc herniation were excluded.

Results. Complications occurring in 7 cases $(9.6 \%)$

Recibido: 9-07-04. Aceptado: 25-10-04 were 2 deep infections, 1 dural tear which was repaired, 2 cerebrospinal fluid fistulae, 1 pseudomeningocele and 1 lesion of $\mathrm{L} 5$ root.

Conclusions.- The most frequent complications were dural tear and infection. These observations are similar to those reported in previously published papers

KEY WORDS: Lumbar discectomy. Complications. Spinal surgery.

\section{Introducción}

La cirugía por hernia discal lumbar es uno de los procedimientos más frecuentes en la práctica neuroquirúrgica $^{11,22,40}$.

En Estados Unidos se realizan 250.000 discectomías de promedio por año ${ }^{10}$. Entre 5 y $20 \%$ de estos pacientes, no mejoran luego de la cirugía, y son varios los factores involucrados ${ }^{15}$.

Entre las principales causas de fracaso de esta cirugía están la incorrecta selección de pacientes y la elección incorrecta del procedimiento quirúrgico ${ }^{10,27}$.

Otro factor que puede marcar la mala evolución de estos pacientes, si bien su frecuencia es baja, es la aparición de complicaciones postoperatorias.

En el presente trabajo se analizan los casos de hernia discal lumbar operados en el Servicio de Neurocirugía del Hospital de Clínicas, Facultad de Medicina, de Montevideo, (Uruguay), entre los años 1997 y 2002, fijando como objetivo identificar los casos en que se presentaron complicaciones, analizar cuales fueron y su frecuencia, plantear métodos que ayuden a prevenirlas, y definir su tratamiento, a la vez que se realiza una revisión bibliográfica en relación con el tema.

\section{Pacientes y métodos}

En el presente trabajo se realizó un estudio retrospectivo, descriptivo, recabando datos de las historias clínicas del Archivo del Hospital Universitario entre los años 1997 y 2002 . 
El criterio de inclusión fue: pacientes que consultaron por un cuadro clínico de compresión radicular, que presentaron cruralgia o ciatalgia, que no se alivió con el trata miento médico, pudiendo presentar además déficit motor y/o sensitivo, y/o disminución o abolición de reflejos osteotendinosos, en los que se realizó el diagnóstico de hernia discal lumbar por tomografía computada (TC) y/o resonancia magnética (RM), cuya topografía fue concordante con la sintomatología, y que fueron tratados quirúrgicamente. No se incluyeron los pacientes con diagnóstico de compresión radicular por estenosis del conducto raquídeo, sin hernia discal.

El total de pacientes con estas características fue de 82. Descartamos 18 casos en los que, por motivos ajenos a nuestra voluntad, no tuvimos acceso a la historia clínica.

Por tanto, la población total para el presente trabajo es de 64 pacientes, 25 de sexo masculino (39\%) y 39 de sexo femenino (61\%).

El promedio de edades de la población estudiada fue de 41 años, con una máxima de 64 años y una mínima de 18 años.

El total de procedimientos quirúrgicos en el período de tiempo definido fue de 75 , incluyendo 8 reintervenciones precoces y 3 reintervenciones diferidas.

Las reintervenciones precoces fueron entre 24 horas y dos meses de la cirugía inicial, y se debieron en 3 casos a error del espacio discal operado, en 3 casos a persistencia de material discal comprimiendo la raíz, un caso para reparar la duramadre por una fístula de LCR, y un caso de limpieza quirúrgica por infección. Las reintervenciones diferidas fueron de 2 casos al año y en un caso a los dos años, y todas se debieron a recidiva herniaria en el mismo espacio discal y del mismo lado.

En los casos en que reaparecieron los síntomas en forma diferida, pero sin clara evidencia de recidiva herniaria en los estudios imaginológicos, se planteó el diagnóstico de fibrosis epidural, y se descartaron como candidatos para cirugía.

La frecuencia de procedimientos en relación a la topografía del disco afectado fue: L3-L4 en 2 casos; L4-L5 en 31 casos; L5-S1 en 31 casos.

\section{Técnica quirúrgica}

Todos los pacientes se operaron en decúbito prono, con el tórax y el abdomen apoyados sobre rollos.

La incisión cutánea fue en línea medía en todos los casos, centrada en el espacio a abordar. Luego se procedió a la desinserción de los músculos del lado afecto.

Catorce pacientes $(21,8 \%)$ presentaban estrechez del neuroforamen, además de una hernia discal, por lo que en
Tabla 1

Frecuencia de las complicaciones observadas (FR (\%): frecuencia relativa porcentual). $\mathrm{N}=\mathbf{7 3}$.

\begin{tabular}{|l|c|r|}
\hline & $\mathrm{N}^{\mathrm{o}}$ de casos & FR (\%) \\
\hline Infección & 2 & 2,7 \\
\hline & & \\
\hline Desgarro dural reparado & 1 & 1,4 \\
\hline Fístula de LCR & 2 & 2,7 \\
\hline Pseudomeningocele & 1 & 1,4 \\
\hline & & 1,4 \\
\hline Lesión de la raíz & 1 & 9,6 \\
\hline TOTAL & 7 & \\
\hline
\end{tabular}

esos casos se realizó foraminotomía más discectomía. En los restantes se realizó hemilaminectomía y discectomía.

Se utilizó técnica microquirúrgica en 51 cirugías $(68 \%)$.

En un solo paciente se realizó la discectomía por la axila de la raíz, maniobra que siempre se intenta evitar y se realiza sólo de necesidad, dado el riesgo de lesión de la raíz o de la duramadre.

\section{Resultados}

Del total de 75 cirugías tendremos en cuenta, para el análisis de las complicaciones, los procedimientos realizados por compresión radicular. Por tanto, excluimos dos de las reintervenciones: una limpieza quirúrgica por infección, y otra para reparar la duramadre por fístula de LCR.

Se presentaron complicaciones en el $9.6 \%$ de las discectomías: 2 casos de infección profunda, 1 caso de desgarro de la duramadre reparado durante la cirugía, 2 casos de fístula de LCR, 1 caso de pseudomeningocele, y 1 caso de lesión parcial de la raíz L5 (Tabla 1).

Este último fue el único paciente que quedó con secuelas secundarias a la cirugía, dado que al momento de alta tenía una leve paresia de la flexión del pie e hipoestesia en el territorio cutáneo de la raíz L5. Esta fue la única complicación que se presentó en el grupo de cirugías en que no se utilizó el microscopio.

No hubo muertes en esta serie.

Ninguna de las complicaciones mencionadas se presentaron en las 3 reintervenciones por recidiva herniaria.

\section{Discusión}

Como se mencionó al inicio, un porcentaje variable de los pacientes operados de una hernia discal lumbar no mejoran su sintomatología o recaen en la evolución. Las 
causas son muy variadas y en conjunto constituyen la etiología del llamado síndrome de fallo de la cirugía espinal lumbar.

Entre las principales causas de fallo de la cirugía podemos citar: a) incorrecta selección de pacientes; b) procedimiento quirúrgico incorrecto; c) asociación de hernia discal con otras lesiones degenerativas del raquis; d) fibrosis epidural y aracnoiditis; e) recidiva herniaria; f) complicaciones.

En relación al último punto, se han reportado en la literatura varios tipos de complicaciones vinculadas directamente al procedimiento.

En las series más grandes analizadas, la frecuencia de complicaciones quirúrgicas fue de entre 2,9 y 10,8\% ${ }^{11,39,40}$.

Son muchas las consecuencias producidas por las complicaciones de la cirugía de hernia discal lumbar, entre las que se cuenta el fracaso de la cirugía, secuelas motoras o sensitivas, o, en raros casos, lesión vascular retroperitoneal, en los que se puede poner en riesgo la vida del paciente.

Las complicaciones se pueden producir y diagnosticar durante la cirugía u objetivarse en el período postoperatorio.

Las complicaciones intraoperatorias descritas son: desgarro de la duramadre $6,8,17,42,49$ lesión de estructuras retroperitoneales ${ }^{5,19,21,24,25,27,33,37,39,40,45,46}$ y lesión de una raíz raquídea ${ }^{10,11,40}$.

Las complicaciones postoperatorias descritas, vinculadas directamente al procedimiento son: infecciones $^{3,4,7,11,14,26,28,29,30,36,39,41,43}$, fístula de $\mathrm{LCR}^{12,22}$, pseu-

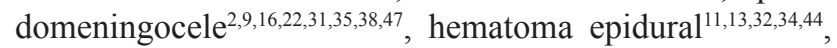
síndrome de Ogilvie ${ }^{1,18,48}$, y síndrome de cola de caballo postoperatorio ${ }^{11,25,40}$ (Tablas 2 y 3 ).

Entre las complicaciones descritas, las más frecuentes son las infecciones, seguidas por el desgarro quirúrgico de la duramadre con sus dos consecuencias: la fístula de LCR o el pseudomeningocele.

\section{Complicaciones Intraoperatorias}

\section{Desgarro de la duramadre}

El desgarro de la duramadre es una complicación que sucede con relativa frecuencia en la cirugía espinal lumbar, y puede advertirse durante la cirugía, [lo que obliga a su reparación, como sucedió en un caso de la presente serie $(1,4 \%)]$, o puede pasar desapercibida ${ }^{49}$. Es en esta última situación en que se convierte en un gran problema, dadas las dificultades que muchas veces se presentan para su resolución.

Es reconocido que el riesgo de desgarro dural es mayor si se trata de una reintervención en un mismo espacio discal. Se ha descrito una incidencia de desgarro dural en
Tabla 2

Complicaciones de la discectomía lumbar

\begin{tabular}{|l|l|}
\hline Complicaciones & \\
\hline Intraoperatorias & Desgarro dural \\
\hline & Incursión en retroperitoneo \\
\hline & Lesión de raíz \\
\hline Posoperatorias & Infecciones \\
\hline & Fístula de LCR \\
\hline & Pseudomeningocele \\
\hline & Hematoma epidural \\
\hline & Síndrome de Ogilvie \\
\hline & Síndrome de cauda equina \\
\hline
\end{tabular}

Tabla 3

Frecuencia de las complicaciones de la discectomía lumbar descritas en la literatura

\begin{tabular}{|l|c|}
\hline Complicaciones $^{11,39,40}$ & $3-11 \%$ \\
\hline Desgarro dural $^{8}$ & $1-3,1 \%$ \\
\hline Lesión retroperitoneal $^{27}$ & $0,50 \%$ \\
\hline Lesión de raíz $^{40}$ & $0,20 \%$ \\
\hline Infecciones $^{11,39}$ & $2-7 \%$ \\
\hline Pseudomeningocele $^{38}$ & $0,3-5 \%$ \\
\hline Hematoma epidural $^{11,34}$ & $0,10 \%$ \\
\hline Síndrome de cauda equina $^{11,40}$ & $0,2-0,8 \%$ \\
\hline
\end{tabular}

la cirugía inicial de 1 a 3,1\%, la cual asciende a 8,1\% para las reintervenciones ${ }^{8}$.

Si la lesión está en la cara posterior del saco dural, en general se puede solucionar con una sutura. Si es lateral o anterior, la solución planteada, con buenos resultados, es sellarla con adhesivos biológicos como el Tissucol $\AA$, o utilizar injertos de tejido adiposo ${ }^{6,17,42}$. A su vez, en estos trabajos se sugiere utilizar las técnicas antes mencionadas, aunque no se objetive salida de LCR, por la eventualidad de desgarro dural inadvertido.

\section{Lesión de estructuras retroperitoneales}

Las estructuras que se pueden lesionar son: vasos retroperitoneales, vísceras o un uréter. Dicha lesión se produce como consecuencia de la perforación del sector anterior del 
anillo fibroso y del ligamento vertebral común anterior, durante una maniobra poco cuidadosa al realizar el curetaje del espacio discal, o menos frecuentemente con la pinza de disco.

La lesión de vasos retroperitoneales, si bien es muy poco frecuente (menor al $0.5 \%)^{27}$, constituye la complicación más grave de la cirugía de hernia discal lumbar, dado que tiene una alta tasa de mortalidad ${ }^{21,24,45}$.

La arteria habitualmente lesionada es la ilíaca primitiva izquierda ${ }^{5,21,24,39,40,45}$, pero también están descritas la lesión de la aorta, vena cava inferior, e inclusive de alguna rama visceral, como por ejemplo la arteria rectal superior ${ }^{46}$.

La lesión de la aorta conlleva una mortalidad de entre 57 y $78 \% \%^{21}$.

$\mathrm{Si}$ la lesión involucra la totalidad de la pared arterial, se puede evidenciar esta complicación durante la cirugía, ya sea al objetivarse una hemorragia copiosa e incoercible a través del espacio discal o, si no es así, que el anestesista note una inestabilidad hemodinámica inesperada y no explicable por otra causa ${ }^{45}$. También debe sospecharse una lesión vascular retroperitoneal, si se evidencia hipotensión arterial en las primeras horas del postoperatorio ${ }^{5,33}$.

Si la lesión no involucra la totalidad de la pared vascular, puede producirse en la evolución, una fístula arterio-venosa o un pseudoaneurisma ${ }^{21,37,40,45}$. La lesión visceral, en general, no se sospecha durante la cirugía, y se plantea varios días después, por la aparición de síntomas de irritación peritoneal, distensión o dolor abdominal y/o fiebre. La TC abdominal evidencia la presencia de aire en la cavidad peritoneal, o en algunos casos abscesos abdominales o pélvicos ${ }^{21}$.

El diagnóstico de lesión del uréter es más difícil por la carencia de síntomas específicos, lo que retrasa el diagnóstico.

En algunos casos se logra diagnosticar porque se asocia a una lesión vascular, la cual es clínicamente evidente ${ }^{19}$. En otros casos se sospecha por un cuadro abdominal inespecífico, con análisis de orina alterado ${ }^{21}$. La realización de una urografía de excreción evidenciará la extravasación del contraste en el sitio de la lesión, o la realizacion de una TC abdominal que pondrá en evidencia una colección retroperitoneal, la cual se realza tardíamente, luego de la inyección de contraste intravenoso ${ }^{20}$.

\section{Lesión de una raíz raquídea}

Es una complicación poco frecuente $y$, realizando una disección cuidadosa, se puede evitar. En la serie de Ramírez y Thisted, se reporta una incidencia de lesión de una raíz raquídea de 2.3 en $10.000^{40}$

En general, la lesión de la raíz se produce por tracción excesiva de la misma durante la discectomía ${ }^{10,11}$, pero también puede ocurrir por no identificarla adecuadamente al momento de incidir con el bisturí el ligamento vertebral común posterior para abordar el espacio discal, la cual se asocia, dado que se lesiona también la duramadre, a fístula de LCR, lo que obliga a reparar el desgarro dural. Este último fue el mecanismo por el cual se produjo la lesión radicular en un caso en la presente serie $(1,4 \%)$. En esa cirugía no se utilizó el microscopio quirúrgico. El uso del microscopio es de gran importancia, ya que no sólo aporta magnificación, sino también una iluminación adecuada.

Esta complicación se asocia a la aparición de dolor neuropático en el postoperatorio.

\section{Complicaciones postoperatorias}

\section{Infecciones}

Constituye la complicación más frecuente de este tipo de cirugía.

La infección se cataloga de superficial o profunda según su relación con la aponeurosis muscular.

La infección superficial involucra el tejido celular subcutáneo y la piel, mientras que las infecciones profundas pueden involucrar el plano muscular, el espacio epidural, o el espacio intervertebral y los cuerpos vertebrales adyacentes constituyendo una espondilodiscitis.

La infección superficial es la complicación postoperatoria más frecuentemente descrita.

En una serie de 654 casos en la que se presentaron complicaciones en 63 pacientes, 45 de estas (71\%) fueron infecciones superficiales, y describen 5 casos de infección profunda $^{39}$. El porcentaje de infecciones en el total de pacientes operados fue de $7,6 \%$

En otra serie, donde se analizaron 984 casos, se constataron complicaciones en 40 pacientes, 23 de las cuales (57\%) fueron infecciones superficiales y 2 casos fueron infecciones profundas ${ }^{11}$. El porcentaje de infecciones en esta serie, sobre el total de cirugías, fue de $2.5 \%$.

Analizamos dos series similares a la nuestra en cantidad de casos y sus resultados fueron: en una serie de 68 casos, hubo dos infecciones superficiales $(2,9 \%)^{43}$, y en la otra, con 100 casos, no tuvieron infecciones ${ }^{28}$.

En la presente serie, la frecuencia de infecciones fue de $2,7 \%$ (2 casos) y, llamativamente, no se observaron infecciones superficiales. Ambos casos presentaron infección de los planos musculares, que evolucionaron favorablemente con tratamiento antibiótico. En uno de los casos fue necesario realizar, además, un debridamiento quirúrgico. Los gérmenes involucrados fueron Stafilococus epidermidis y Stafilococus aureus.

Una situación poco frecuente, pero de graves consecuencias, es la coexistencia de un proceso infeccioso postoperatorio y una fístula de LCR, ya que la complicación más temible es la meningitis. 
El mecanismo por el cual se produce esta doble complicación es secuencial, dado que la fístula de LCR impide que la herida operatoria cicatrice y esto crea las condiciones para que se colonice por gérmenes.

Si se plantea la realización de una limpieza quirúrgica, se puede intentar reparar la fístula en el mismo procedimiento, concomitante con el tratamiento antibiótico adecuado al germen implicado.

Numerosos trabajos concluyen que el uso de una dosis profiláctica de antibióticos, en la hora previa del comienzo de una cirugía, disminuye el índice de infecciones asociadas a la misma $3,4,4,14,23,26,29$.

Se recomienda, en general, fármacos que cubran la flora cutánea, siendo las cefalosporinas de primera generación las más comúnmente utilizadas y probadas, dado que son efectivas frente a Stafilococus aureus y epidermidis, patógenos habitualmente implicados en infecciones en cirugía espinal, y cubren también Escherichia coli y proteus, patógenos que también pueden estar involucrados en algunas ocasiones ${ }^{14}$.

Se han reportado casos de gérmenes menos comunes como Serratia ${ }^{23}$ o aspergillus $^{36}$.

En la serie de Ramírez y Thisted, en la que se analizaron las complicaciones de la cirugía de hernia discal lumbar, en un período de un año en varios centros de Estados Unidos, se constató una incidencia de infecciones de 30,7 por 10.000 , y de éstas, en el $70 \%$ el germen implicado fue Stafilococus y en el 16\% gérmenes Gram negativos ${ }^{40}$.

Dentro del grupo de las cefalosporinas, la cefazolina, es uno de los fármacos más utilizados para profilaxis quirúrgica $^{4,14}$.

Con una dosis de 2 gramos intravenosos de cefazolina se logran concentraciones inhibitorias mínimas en suero durante 12 horas, y en el LCR durante 5 horas $^{29}$ e, inclusive, se comprobaron niveles detectables del fármaco dentro del disco intervertebral por lapsos que varían entre 15 y 80 minutos ${ }^{7,41}$.

Se han probado otros antibióticos, como cefoperazona y sulbactam, detectándose los mismos a concentraciones inhibitorias mínimas en el disco intervertebra ${ }^{30}$.

\section{Fístula de LCR}

Como ya se mencionó, el desgarro de la duramadre es una complicación que sucede con relativa frecuencia en la cirugía espinal lumbar.

Si una lesión dural se hace evidente en el postoperatorio inmediato, al objetivarse una fístula de LCR, ésta se debe solucionar rápidamente, dado que, como ya mencionamos, la salida de líquido retrasa la cicatrización de la herida operatoria y predispone a una infección con graves consecuencias.

En general, esta complicación se resuelve con medidas conservadoras, tales como dejar al paciente en posición horizontal, realizar punciones lumbares evacuadoras o colocar un drenaje lumbar. Pero ante el fracaso de estas medidas puede ser necesaria una reintervención para intentar localizar y reparar el desgarro dural.

En el presente estudio se presentó esta complicación en dos casos $(2,7 \%)$. En uno mejoró con el tratamiento conservador. El segundo requirió de una reintervención para reparar el desgarro dural.

Se han reportado buenos resultados ante fístulas de LCR de difícil resolución, y una vez agotadas todas las medidas terapéuticas, mediante la realización de una derivación lumbo peritoneal ${ }^{12}$.

El riesgo del tratamiento conservador es que se logre una mejoría transitoria y, meses o años más tarde, el paciente consulte por un pseudomeningocele ${ }^{22}$.

\section{Pseudomeningocele}

El líquido cefalorraquídeo que fluye a través de un desgarro dural inadvertido ocupa la cavidad virtual o real resultante de la disección quirúrgica y, lentamente, la va rellenando formando lo que se denomina un pseudomeningocele. Si el desgarro no involucra la aracnoides, se puede producir un quiste aracnoideo extradural ${ }^{9,22}$.

La incidencia del pseudomeningocele es de 0,3 a $5 \%{ }^{38}$. Frecuentemente el pseudomeningocele cursa en forma asintomática, y puede diagnosticarse por controles imaginológicos realizados por otras causas. Su incidencia, por tanto, debe ser mayor de la establecida. En estos casos se resuelve espontáneamente debido, seguramente, a que el LCR sale a muy baja presión, y el desgarro aracnoido-dural cicatriza9.

El pseudomeningocele sintomático se puede diagnosticar pocas semanas después de la cirugía, dado que el paciente se queja de dolor constante en la herida operatoria y se puede notar hinchazón a nivel de la cicatriz. En algunos casos reaparece el dolor radicular. Puede acompañarse de cefalea debida a hipotensión endocraneana generada por la disminución del volumen de LCR. El diagnóstico se realiza fácilmente mediante la TC del raquis, donde se visualiza una colección con densidad de líquido, que se origina en la laminectomía y que abarca en una extensión variable los planos musculares, llegando inclusive hasta el tejido subcutáneo.

Si el pseudomeningocele es pequeño y asintomático, en general se resuelve con medidas conservadoras, como ya se discutió para los casos de la fístula de LCR. Sin embargo, si la colección es grande y genera dolor, la solución es la reparación quirúrgica del desgarro dural, que en los casos de diagnóstico precoz suele ser pequeño ${ }^{16}$.

Pero a diferencia de los casos anteriores, si el diagnóstico de pseudomeningocele se realiza meses o años 
después de la intervención, la resolución quirúrgica puede ser más difícil, dado que el defecto dural suele ser grande e inclusive puede tener raíces raquídeas en su interior, siendo el motivo de consulta síntomas de compresión radicular, muchas veces graves ${ }^{2,22,35,47}$.

El objetivo de la intervención es liberar la raíces y reparar el defecto dural. Si el defecto no permite un cierre simple mediante una sutura, se debe colocar un injerto, suturarlo y sellar con adhesivos biológicos $22,38,47$.

En la presente serie se presento un solo caso de pseudomeningocele $(1,4 \%)$, el cual se diagnosticó por TC, realizada por la reaparición precoz de lumbalgia asociada a cefalea. Desconocemos la evolución de esta paciente dado que, luego del diagnóstico, no se controló más en el Servicio de Neurocirugía del Hospital Universitario.

Se han descrito raras complicaciones asociadas al desgarro dural tales como herniación y atrapamiento de raíces raquídeas en el espacio discal ${ }^{31}$, o pseudomeningocele calcificado y sintomático, diagnosticado 6,5 años después de la cirugía ${ }^{47}$.

\section{Hematoma epidural}

El hematoma epidural como complicación posterior a la cirugía espinal, que cause nuevo déficit neurológico postoperatorio, es muy raro $^{13,32}$.

Lawton $^{34}$, y Davis ${ }^{11}$ reportan una incidencia de $0,1 \%$. Debemos sospechar que podemos estar ante esta complicación, cuando en las primeras horas de postoperatorio reaparece el dolor radicular, o parestesias, acompañado por déficit motor, que en general es bilateral, aunque se haya abordado de un solo lado ${ }^{13,32,44}$.

No se han identificado claramente factores predisponentes para la aparición de un hematoma extradural espinal postoperatorio. En un trabajo se identificaron, como posibles factores de riesgo de esta complicación, la laminectomía de más de un espacio y la presencia de una coagulopatía ${ }^{32}$.

Ante la aparición del cuadro clínico descrito en el postoperatorio inmediato, se debe estudiar rápidamente el paciente con TC o RM para confirmar el diagnóstico y reintervenirlo de urgencia. Actuando de esta manera, la posibilidad de recuperación sin secuelas es muy alta ${ }^{32,44}$.

\section{Sindrome de Ogilvie}

El Síndrome de Ogilvie o pseudo-obstrucción del colon, es una complicación muy rara de la cirugía espinal lumbar, y constituye un diagnóstico diferencial con la lesión visceral.

Se produce una gran dilatación cecal, sin evidencia de obstrucción mecánica, que debe ser tratado rápidamente, dado el riesgo de perforación del ciego. Su etiopatogenia no es muy clara, pero podría estar relacionada a una alteración de la inervación vegetativa del colon secundaria a la cirugía raquídea, por un disbalance entre la inervación simpática y parasimpática.

Se ha sugerido una vinculación con la tracción excesiva y mantenida del saco dural durante una discectomía dificultosa $^{48}$.

El paciente presenta distensión abdominal, dolor difuso, ausencia de ruidos, hidroaéreos, y detención del tránsito digestivo sin expulsión de materias ni gases. En la radiografía de abdomen se puede visualizar una dilatación proximal del colon, sobre todo a nivel del ciego.

Si bien se han descrito procedimientos quirúrgicos para tratar esta entidad, como la cecostomía, o la degravitación por vía endoscópica, recientemente se lograron buenos resultados con la neostigmina, inhibidor de la colineste$\operatorname{rasa}^{1,18,48}$.

\section{Sindrome de cola de caballo postoperatorio}

Constituye otra rara complicación de la cirugía del raquis lumbar. Davis reporta una incidencia de $0.2 \%{ }^{11}$. En la serie de Ramírez y Thisted la incidencia de esta complicación fue de 8.2 por $10.000^{40}$.

Los signos aparecen precozmente luego de la cirugía, lo que obliga a estudiar al enfermo para valorar la causa, no encontrándose en general compresión mecánica de las raíces.

Su fisiopatología no es clara, pero, en los casos reportados, aparece como complicación de la discectomía lumbar en pacientes con estenosis del canal raquídeo. Esto ha llevado a pensar en la estasis venosa como causa de este evento $^{25}$. También se plantea que esta complicación pueda ser secundaria a la tracción prolongada de las raíces, lo que puede generar edema de las mismas ${ }^{11}$.

El tratamiento es la laminectomía de urgencia, con lo cual se logra una buena recuperación ${ }^{25}$.

\section{Conclusiones}

Como vimos, la frecuencia de complicaciones en las series analizadas fue de aproximadamente 3 a $11 \%$, siendo de $9.6 \%$ en el presente estudio.

Como se mencionó al inicio, las infecciones y el desgarro de la duramadre son las complicaciones más frecuentes, resultado que se repite en la presente serie.

Se observaron $2,7 \%$ de infecciones, lo cual constituye una frecuencia baja de esta temida complicación. Sin embargo, la frecuencia de desgarro dural fue superior a la de otras series: $5,5 \%$, donde incluimos desgarro reparado $(1,4 \%)$, fístula de LCR $(2,7 \%)$ y pseudomeningocele $(1,4 \%)$.

Se observó un solo caso de lesión de una raíz $(1,4 \%)$, 
la cual pudo haberse evitado con el uso del microscopio quirúrgico.

Como ya mencionamos al principio, la discectomía lumbar es una cirugía con una baja incidencia de complicaciones. Pero, aun así, debe tener presente que existen y que pueden llegar a ser graves, por lo que el cirujano debe estar atento a la evolución del paciente, para diagnosticarlas y tratarlas en forma adecuada.

\section{Bibliografía}

1. Althausen, P., Gupta, M., Benson, D., Daniel, R., Jones, D.: The use of neostigmine to treat postoperative ileus in orthopedics spinal patients. J Spinal Disorders 2001; 14: 541545 .

2. Amaolo, M., Palini, S., Passante, V., Desole, D., Olivetti, M., González, J.: Pseudomeningocele postcirugía de la columna lumbar. Comunicación de dos casos. Revista Argentina de Neurocirugía 2003; 17: 197-200.

3. Barker, F.G. $2^{\circ} .:$ Efficacy of prophylactic antibiotic therapy in spinal surgery: a meta analysis. Neurosurgery 2002: 5: 391-400.

4. Beiner, J., Grauer, J., Kwon, B., Vaccaro, A.: Postoperative wound infections of the spine. Neurosurg Focus 2003; 15 : article 14 .

5. Bilbao, G., Menchacatorre, I., Uriguen, M., et al.: Complicaciones intraabdominales de la cirugía de la hernia discal lumbar. Neurocirugía 2004; 15: 279-284.

6. Black, P.: Cerebrospinal fluids leaks following spinal surgery: use of fat grafts for prevention and repair. Technical note. J Neurosurg 2002 (Spine 2); 96: 250-252.

7. Boscardin, J.B., Ringus, J.C., Feingold, D.J., Ruda, S.C.: Human intradiscal levels with cefazolina. Spine 1992; 17 (6 suppl.): 5145-5148.

8. Cammisa ,F.P., Jr, Girardi, F.P., Sangani, P.K., et al.: Incidental durotomy in spine surgery. Spine 2000; 25: 26632667.

9. Couture, D., Branch, Ch.: Spinal pseudomeningoceles and cerebrospinal fluids fistulas. Neurosurg Focus 2003: 15 article 6 .

10. Cybulski,, G.: Evaluation and management of epidural fibrosis and adhesive arachnoiditis in failed lumbar spine surgery. In Tindall, G., Cooper, P., Barrow, D., eds. The Practice of Neurosurgery. Baltimore, 1996: 2565-2573.

11. Davis, R.: A long-term outcome analysis of 984 surgically treated herniated lumbar disc. J Neurosurg, 1994 80: 415-421.

12. Deen, H.G., Pettit, P.D., Sevin, B.U., Wharen, R.E., Reimer, R.: Lumbar peritoneal shunting with video-lapa roscopic assistance: a useful technique for the management of refractory postoperative lumbar CSF leaks. Surg Neurol 2003; 59: 473-477.

13. Di Lauro, L., Poli, R., Bortoluzzi, M., Marini, G.:
Parestesias after lumbar disc removal and their relationship to epidural hematoma. J Neurosurg 1982-57: 135-136.

14. Dimick, J.B., Lipsett, P.A., Kostuik, J.: Antimicrobial prophilaxis in spine surgery: basic principies and recent advances. Spine 2000; 25: 2544-2548.

15. Ebeling, U., Kalbarcik, H., Reulen, H.: Micosurgical reoperation following lumbar disc surgery. J Neurosurg 1989; 70: 397-404.

16. Eichholz, K., Ryken, T.: Complications of revision spinal surgery. Neurosurg Focus 2003; 15: article 1.

17. Eismont, F.J., Wiesel, S.W., Rothman, R.H.: Treatment of dural tears associated with spinal surgery. J Bone Joint Surg 1981; 63: 1132-1136.

18. Feldman, R., Karl, R.: Diagnosis and treatment of Ogilvie's syndrome after lumbar spinal surgery. J Neurosurg 1992; 76: 1012-1016.

19. Gangai, M.: Ureteral injury incident to lumbar disc surgery. J Neurosurg 1972; 36: 90-92.

20. Gayer, G., Caspi, I., Garniek, A., Hertz, M., Apter, S.: Perirectal urinoma from ureteral injury incurred during spinal surgery mimicking rectal perforation on computed tomography scan. Spine 2002; 27: E451-453.

21. Googkin, R., Laska, L.: Vascular and visceral injuries associated with lumbar disc surgery: medicolegal implications. Surg Neurol 1998; 49: 358-372.

22. Hadani, M., Findler, G., Knoler, N., Tadmor, R., Sahar, A., Shacked, I.: Entrapped lumbar nerve root in pseudomeningocele after laminectomy: report of three cases. Neurosurgery, 1986: 19: 405-407.

23. Hadjipavlou, A.G., Gaitanis, I.N., Papadopoulos, C.A., Katonis, P.G., Konta, G.M.: Serratia spondylodiscitis after elective lumbar spine surgery: report of two cases. Spine 2002; 27(2P): E507-512.

24. Harrington, J.F. Jr.: Far lateral disc excision at L5 S1 complicated by iliolumbar artery incursion: case report. Neurosurgery $2001 ; 48$ : 1377-1380.

25. Henriques, T., Olerud, C., Petren-Mallmin, M., Ahl, T.: Cauda equina syndrome as a postoperative complication in five patients operated for lumbar disc herniation. Spine 2001; 26: 293-297.

26. Horwitz, N.H., Curtin, J.A.: Prophylactic antibiotics and wound infections following laminectomy for lumbar disc herniation. J Neurosurg 1975; 43: 727-731.

27. Iglesias, P., Ruiz, P., Alday, R., De la Cruz, J., Diez R.: Evaluación del proceso quirúrgico de la hernia discal lumbar II. Una aproximación a la calidad científico técnica. Neurocirugía 2001;12: 429-438.

28. Jeffrey Lewis, P., Weir, B., Broad, R., Grace, M.: Long-term prospective study of lumbosacral discectomy. J Neurosurg 1987; 67: 49-53.

29. Klekner, A., Ga'spa'r, A., Kardos, S., Szabo, J., Cse'csei, G.: Cefazolin prophylaxis in neurosurgery monitored by capillary electrophoresis. J Neurosurg Anesthesiol 2003; 
150: 249-254.

30. Köroglu, A., Acar, O., Üstün, M.E., Tras, B., Eser, O.: The penetration of cefoperazone and sulbactam into the lumbar intervertebral disc. J Spinal Disorders 2001; 14: 453454.

31. Kothbauer, K., Seiler, R.: Transdural cauda equina incarceration after microsurgical lumbar discectomy: case report. Neurosurgery 2000; 47: 1449-1451.

32. Kou, J., Fischgrund, J., Biddinger, A., Herkowitz, H.: Risk factors for spinal epidural hematoma after spinal surgery. Spine 2002; 27: 1670-1673.

33. Lange, M., Fink, U., Philipp, A., Oeckler, R.: Emergency diagnosis with spiral CT angiography in case of suspected ventral perforation following lumbar disc surgery. Surg Neurol 2002; 57: 15-19.

34. Lawton, M.T., Porter, R.W., Heiserman, S.E. et al.: Surgical management of spinal epidural hematoma: relationship between surgical timing and neurological outcome. J Neurosurg 1995; 83: 1-7.

35. Lee, K.S., Hardy, I.M.: Postlaminectomy lumbar pseudomeningocele: report of four cases. Neurosurgery 1992 , 30: 111-114.

36. Mawk, J.R., Erickson, D.L., Chou, S.N., Seljeskog, E.L.: Aspergillus infections of de lumbar disc spaces. J Neurosurg 1983; 58: 270-274.

37. May, A.R., Brewster, D.C., Darling, R.C., Browse, N.L.: Arteriovenous fistula following lumbar disc surgery. $\mathrm{Br}$ J Surg 1981; 68: 41-43.

38. Misra, S., Morgan, H., Sedler, R.: Lumbar myofascial flap for pseudomeningocele repair. Neurosurg Focus 2003; 15 : article 13.

39. Pappas, C.T., Harrington, H., Sonntag, U.K.: Outcome analysis in 654 surgically treated lumbar disc herniations. Neurosurgery, 1992: 30: 862-866.

40. Ramírez, L., Thisted, R.: Complications and demographic characteristics of patients undergoing lumbar discectomy in community hospitals. Neurosurgery, 1989: 25: $226-231$.
41. Rhoten, P., Murphy, M., Kalfas, I., Hahn, J., Washington, J.: Antibiotic penetration into cervical discs. Neurosurgery 1995; 37: 418-421.

42. Robertson, J., Soble, J., Powers, N., Nelson, P.: Prevention of cerebrospinal fluid fistulae and reduction of epidural scar with new surgical hemostat device in a porcine laminectomy model. Spine 2003; 28: 2298-2303.

43. Rogers, L.: Experience with limited versus extensive disc removal in patients undergoing microsurgical operations for ruptured lumbar disc. Neurosurgery 1988; 22: 82-85.

44. Scavarda, D., Peruzzi, P., Bazin, A., Scherpereel, B., Gomis, P., Graftieaux, J.P., et al.: Hematomes extra-duraux rachidiens post-operatoires. Quatorze observations. Neurochirurgie 1997; $43:$ 220-227.

45. Shevlin, W., Luessenhop, A., Fox, J., McCullough, D.: Perforation of the anterior annulus during lumbar discectomy. J Neurosurg 1973; 38: 514-515.

46. Tsai, Y.D., Yu, P.C., Lee, T.C., Chen, H.S., Wang, S.H., Kuo, Y.L.: Superior rectal artery injury following lumbar disc surgery. Case report. J Neurosurg 2001; 95 (1 suppl): 108110 .

47. Tsuji, H., Handa, N., Handa, O., Tajima, G., Mori, K.: Postlaminectomy ossified extradural pseudocyst. J Neurosurg 1990; 73: 785-787.

48. Vega, S.D., Mosquera, G., Varela, A.: Síndrome de Ogilvie. Presentación de 3 casos. Neurocirugía 2002; 13: 229232.

49. Wang, J., Bohiman, H., Riew, D.: Dural tears secondary to operations on the lumbar spine: management and results after a two years minimum folowup of eighty-eight patients. J Bone Joint Surg 1998; 80: 1728-1732.

Hernández-Pérez, P.A.; Prinzo-Yamurri, H.: Análisis de las complicaciones de la cirugía de hernia discal lumbar. Neurocirugía 2005; 16:419-426.

Correspondencia postal: Dr. Pablo Hernández Pérez. Feliciano Rodríguez 3293. 11600 Montevideo. Uruguay. 